\title{
Investigation into mechanical properties to use recycled Polypropylene/Talc composites for car bumper application
}

\author{
Sulove Timsina \\ Mechanical Engineer, Mechanical Section,Office of Municipal Executive,Pokhara Metropolitan City \\ Email:sulovezen@gmail.com
}

\begin{abstract}
In today's modern dynamic world, automobile industry has seen many revolutions and developments over couple of decades to improve the performance and efficiency. One of the major revolutions was to replace metallic components to high performance polymer composites to reduce total weight of automobiles. As a result we can find more than $50 \%$ use of polymer composite and other high performance materials in automobiles like passenger car. In addition to this, the present development is towards making low cost models in cars to make it available to middle class families. Many OEM (Original Equipment Manufacturers) are working for different cost cars for same model to sell across the globe based on the economic status of the countries. Also, unlike metals, which are easily recoverable and recyclable, plastic waste increase forces the government to legislate for the limitation of such waste by introducing the concept of recycling. As a result manufacturers are looking for use of virgin polymer (Polypropylene) along with some percentage of recycled one to reduce the total cost of manufacturing.

The present recycling world uses used materials; reprocess them so that they can be used for further applications. This recycling business emphasizes on quantity rather on quality, so mechanical properties actually changes when product made out of Polypropylene are being recycled. The thing is to see how the mechanical strength changes when Polypropylene is recycled and what are the variations in mechanical properties when unused Polypropylene (PP) is mixed with some percentage of Recycled Polypropylene (RPP) along with some percentage of Impact Modifier and Filler material.
\end{abstract}

\section{KEYWORDS: OEM,PP,RPP}

\section{Introduction}

A polymer is a large molecule, or macromolecule, composed of many repeated subunits. Those subunits can be termed as "monomers". The term "polymer" derives from the ancient Greek word $\pi \mathrm{o} \lambda u ́ \varsigma$ (polus, meaning "many, much") and $\mu$ ćpos (meros, meaning "parts"), and refers to a molecule whose structure is composed of multiple repeating units, from which originates a characteristic of high relative molecular mass and attendant properties. ${ }^{[1]}$ Polymers having wide range of properties, both synthetic 
and natural polymers play an essential role in day-to-day life. One of the polymers we are using in our thesis is Polypropylene.

Polypropylene is a low density thermoplastic, used for its toughness, and fatigue and chemical resistance (Crawford, 1998). Polypropylene is an economical material that offers a combination of outstanding physical, chemical, mechanical, thermal and electrical properties not found in any other thermoplastic. These properties make polypropylene attractive in the automotive industry, including fascias, bumpers and battery casing. Recycled PP is also largely used in the automotive industry (Scheirs, 1998). Generally Polypropylene is used as bumper material in automotive field.

Mechanically Polypropylene (PP) is normally tough and flexible, especially when copolymerized ${ }^{[2]}$ with ethylene. ${ }^{[3]}$ This allows it to be used as an engineering plastic in replacement of Acrylonitrile Butadiene Styrene (ABS).

\section{Recycled Polypropylene (Rpp)}

Recycling has attracted broad interest due to pressure from legislations, saving natural resources, reducing cost, landfill and emission. Regarding emissions, it was estimated that using recycled plastics could reduce greenhouse gas emissions by about $80 \%{ }^{[4]}$

So use of Recycled Polypropylene in various industries has been a trend to reduce cost as well as to cope with the problems regarding pollution. But when compared to virgin plastic, recycled plastic tend to show lesser performance due to degradation phenomena that occur during the product's first life and reprocessing.

The performance and properties of these RPP products can be increased to some extent by using these RPP with virgin PP along with filler material and impact modifier. This forms a Polymer composite. Here in our thesis we are using virgin PP and RPP as matrixes, Ethylene Propylene Diene Monomer (EPDM) as an impact modifier and Magnesium Silicate $\left[\mathrm{Mg}_{3} \mathrm{Si}_{4} \mathrm{O}_{10}(\mathrm{OH})_{2}\right]$ as filler material to make a polymer composite that can be used for automotive applications.

FILLER: TALC (Magnesium Silicate/ calcium Carbonate)

Talc is a clay mineral composed of hydrous magnesium silicate with the chemical formula $\left[\mathrm{H}_{2} \mathrm{Mg}_{3}\left(\mathrm{SiO}_{3}\right)_{4}\right]$ or $\left[\mathrm{Mg}_{3} \mathrm{Si}_{4} \mathrm{O}_{10}(\mathrm{OH})_{2}\right]$. In loose form, it is the widely used substance known as baby powder or talcum powder. It is monoclinic or triclinic crystal system ${ }^{[5]}$. Talc is primarily formed via hydration and carbonation via the following reactions;

Serpentine + Carbon dioxide $\rightarrow$ Talc + Magnesite + Water

$2 \mathrm{Mg}_{3} \mathrm{Si}_{2} \mathrm{O}_{5}(\mathrm{OH}) 4+3 \mathrm{CO}_{2} \rightarrow \mathrm{Mg}_{3} \mathrm{Si}_{4} \mathrm{O}_{10}(\mathrm{OH})_{2}+3 \mathrm{MgCO}_{3}+3 \mathrm{H}_{2} \mathrm{O}$

Talc can also be formed via a reaction between dolomite and silica;

$3 \mathrm{CaMg}\left(\mathrm{CO}_{3}\right)_{2}+4 \mathrm{SiO}_{2}+\mathrm{H}_{2} \mathrm{O} \rightarrow \mathrm{Mg}_{3} \mathrm{Si}_{4} \mathrm{O}_{10}(\mathrm{OH})_{2}+3 \mathrm{CaCO}_{3}+3 \mathrm{CO}_{2}$

The product coming from the dolomite and silica results in magnesium silicate and calcium carbonate. The obtained calcium carbonate can be used as a filler material in car bumper application. The cost of the calcium carbonate is less because it is the by product. But the properties imparted by calcium carbonate is quite good and hence it is utilized in car bumper 
application.

\section{IMPACT MODIFIER: Ethylene Propylene}

\section{Diene monomer}

The main disadvantage of this polymer (PP) is its low temperature impact resistance. To improve impact resistance, blending of ethylene propylene diene monomer (EPDM) with $\mathrm{PP}$ is a well known technique. ${ }^{[6]}$ However, this type of thermoplastic elastomer olefinic (TPO) has an unstable morphology because of coalescence of dispersed rubber particles and low compatibility between rubber phase and thermoplastic matrix. To stabilize the morphology of the blend, the rubber phase is vulcanized in situ-during the melt blending while to improve compatibility and achieve finer dispersion of rubber particles, using appropriate compatibilizer and block or graft copolymer have been reported. ${ }^{[7]}$ this process can improve properties such as oil resistance, tensile properties, maximum service temperature etc.

\section{TYPES OF BUMPERS}

Plastic Bumper:-Most modern car uses a reinforced thermoplastic bumper, as they are cheap to manufacture, easy to fit and absorb more energy during a crash. A majority of car bumpers are custom made for a specified model, so if you are looking to replace a cracked bumper with a similar one, you would have to buy from a specialist dealer. However, many companies now offers alternative design in thermoplastic; with a range of fitting designed for different models. It is easy because plastic does not require better planning to get the proper design but requires best planning for the selection of material and their properties. Nowadays PP is mainly preferred for bumper application and other automotive parts.

1. Boby kit Bumper:-Modified cars often now have a full body kit rather than just a front and rear bumper. These kits acts as a skirt around the entire body of the car and improves the performance by reducing the amount air flowing underneath the car and so reducing drag.

2. Carbon Fiber Bumper:-Carbon fiber body work is normally the thing of super-cars, but many car companies, and specialist modifiers, are starting to use it for replacement body part on everyday cars. This is because it is very light and is safe during a crash. It is, however, a lot more expensive than normal thermoplastic.

3. Steel bumper:-Originally plated steel was used for the entire body of a car, including the bumper. This material worked well, as it was very strong in a crash, but it was very heavy and dented performance. In order to reduce the overall weight of the car and to increase the engine performance, nowadays plastics are preferred.

Various types of bumpers are studied and plastic bumpers are the most used as a car bumper in an automotive industry.

The various grades are available based on their applications. Their families are listed below:-

- Homo Family

- Random copolymer Family

- Impact copolymer Family

Bumpers have shock absorbers on them to absorb the shock of a minor impact (5mph) 
without causing significant damage to the car or the object hit. So, if you backed into a concrete wall while parking, the bumper would move with the shock absorbers- you might get a scuff on the paint; if there was no shock absorber, the bumper or the mounting brackets would get bent (or the wall would break).

They all serve the same function: protecting the occupant of the vehicle from the traumatic shock during a collision or lessening body damage to the vehicle by absorbing collision force. Some bumpers have extremely technical features and designs.

\section{METHODOLOGY}

* Fine strands of polypropylene composite is prepared in twin screw extruder by mixing suitable percentage by weight of VPP, RPP, Talc and Impact modifier

* The variety of specimens required for the particular tests are prepared by using injection molding machine

To test the following properties

1. Mechanical Properties

- Tensile Strength

- Elongation at Break

- Flexural Strength

- Izod Impact Strength (Notched)

2. Thermal Property

- Heat Deflection Temperature (HDT)

* To compare result obtained from the tests with the property of Virgin Polypropylene.

\section{Apparatus Required}

- Mixing Bucket

- Weighing Machine

- Twin screw Extruder
- Liquid Injection Molding Machine

- Tensile Tester Testing Machine

- Impact Testing Machine

- Flexural Tester

- H.D.T. (Hot Deformation) Tester

\section{Materials Used}

- Virgin Polypropylene (VPP)

- Recycled Polypropylene (RPP)

- Talc (Calcium Carbonate)

- Impact Modifier (Ethylene Propylene Diene Monomer)

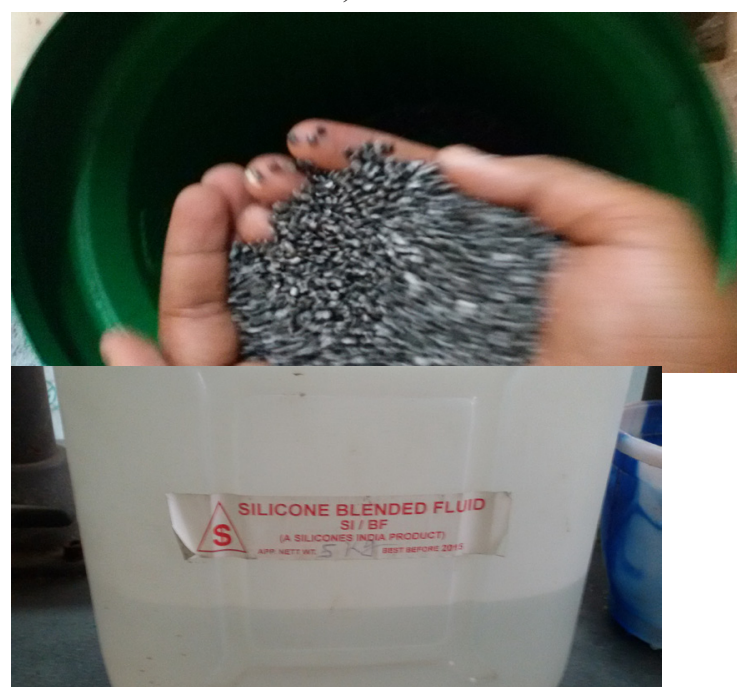

Figure 1:- Bucket and Silicon oil gallon

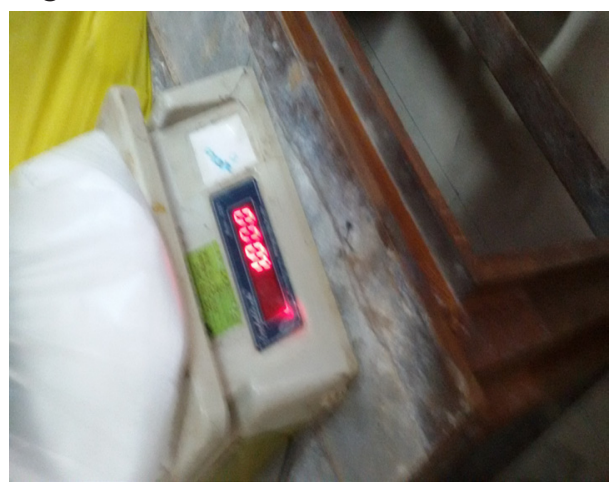

Figure 2:- Weighing Machine

Twin Screw Extruder :- Twin screw extruder is used extensively for mixing, compounding, 

or reacting polymeric materials. The flexibility of twin screw extrusion equipment allows this operation to be designed specifically for the formulation being processed. For example, the two screws may be corotating or counterrotating, intermeshing or nonintermeshing. In addition, the configurations of the screws themselves may be varied using forward conveying elements, reverse conveying elements, kneading blocks, and other design in order to achieve particular mixing characteristics ${ }^{[18]}$.

This process starts by feeding plastic materials (pellets, granules, flakes or powders) from a hopper into the barrel of the extruder. The material is gradually melted by the mechanical energy generated by turning screws and by heaters arranged along the barrel. The molten polymer is then forced into a die, which shapes the polymer into a pipe that hardens during cooling.

At the front of the barrel, the molten plastic leaves the screw and travels through a screen pack to remove any contaminants in the melt. The screen are reinforced by a breaker plate since the pressure at this point exceeds. The breaker plate assembly also serves to create back pressure into the barrel. Back pressure is required for uniform melting and proper mixing of the polymer.

Plastics are very good thermal insulators and are therefore difficult to cool quickly. Compared to steel, plastic conducts its heat away 2000 times more slowly. In a tube or pipe extrusion line, a sealed water bath is acted upon by a carefully controlled vacuum to keep the newly formed and still molten tube or pipe from collapsing.

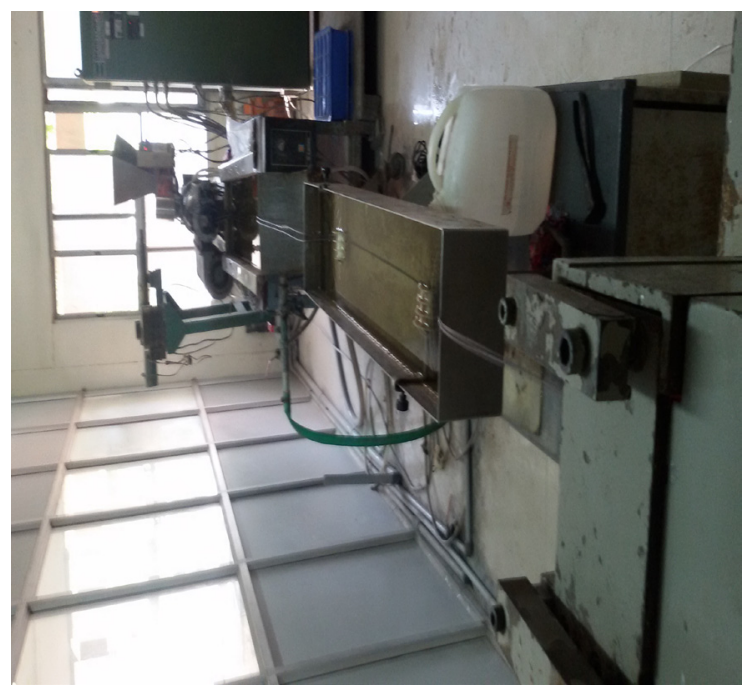

Figure 3:-Twin Screw Extruder

Injection Molding Machine:- An Injection molding machine, also known as an injection process, is a machine for manufacturing plastic products by injection molding process. It consists of two main parts, an injection unit and a clamping unit.

Injection molding machine can fastens the molds in either a horizontal or vertical position. The majority of the machines are horizontally oriented, but vertical machines are used in some niche applications such as insert molding, allowing the machine to take the advantage of gravity. There are many ways to fasten the tools to the platens, the most common being manual clamps (both halves are bolted to the platens); however hydraulic clamps and magnetic clamps are also used. The magnetic and hydraulic clamps are used where fast tool changes are required.

The thermoplastic material is supplied to the molders in the form of small pellets. The hopper on the injection molding machine holds these 
pellets. The pellets are gravity-fed from the hopper through the hopper throat into the barrel and screw assembly. The barrel supports the reciprocating plasticizing screw. It is heated by electric heater bands. The reciprocating screw is used to compress, melt, and convey the material. The reciprocating screw consists of three zones; Feeding Zone, Compressing (or Transition) Zone and Metering Zone.

The feed zone conveys the plastic pellets which are fed from the hopper to the transition zone where they are compressed by a change in screw geometry. This compression forces the pellets to melt through the action of pushing up against each other. This is called shearing. The metering zone that conveys the melt to the front of the screw ready for injection into the mould cavity.

In the transition zone material is compressed by the change in the depth of the screw channels from the feed zone to metering zone. The ratio of change in depth is called the compression ratio and is usually between 2and for plastics such as PP and PE. The length of the transition zone is typically 4 to $7 \mathrm{x}$ the screw diameter in general purpose screw. The temperature ranges from $160^{\circ} \mathrm{C}$ to $320^{\circ} \mathrm{C}$.

The nozzle connects the barrel to the sprue bushing of the mold and forms a seal between the barrel and mold. The temperature of the nozzle should be set to the material's melt temperature or just below it, depending on the recommendation of the material supplier. The control system provides the consistency and repeatability in machine operation. It monitors and control the processing parameters, including the temperature, pressure, injection speed, screw speed and position. The process control has the direct impact on the final product quality.

The clamping system opens and closes the mold, supports and carries the constituent part of the mold, and generates sufficient force to prevent the mold from opening. Clamping force can be generated by a mechanical (toggle) lock, hydraulic lock, or a combination of the two basic types. Finally the molded specimen can be ejected by forcing the ejector pins.

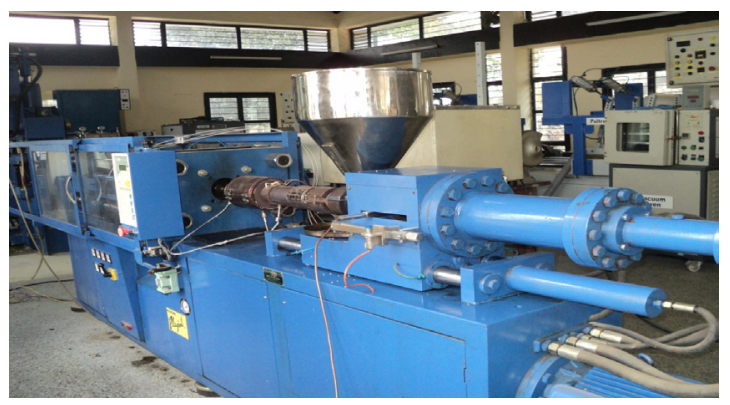

Figure 4:- Injection Molding Machine

Tensile Tester Machine:- ASTM D638 and ISO 527 are technical equivalents. This method is designed to determine the tensile properties of the plastic materials. And can be used for testing any materials of any thickness upto 14 $\mathrm{mm}$. However, it is preferable to use ASTM Test Methods D882 for the specimen of thin sheeting including film less than $1.0 \mathrm{~mm}$. All the materials of thickness greater than $14 \mathrm{~mm}$ must be reduced by machining before this method can be applied.

The Tensile Properties of reinforced and unreinforced plastics may be determined by using standard dumbbell- shaped test specimen tested under defined condition of pretreatment, temperature, humidity, and testing machine 
speed. These properties include true Stress and strain, Engineering Stress and strain, the Elastic Modulus, the Ultimate Tensile Strength, the Fracture Stress, the Modulus or Toughness, and the Modulus of Resilience.

Poisson's ratio at room temperature also may be determined using the ASTM D638 test method. Poisson's ratio is a measure of transverse strain versus axial strain when uniaxial stress is applied.

Poisson's ratio is important when an engineer needs to design for all dimensional changes due to applied forces. Poisson's ratio is also employed when applying the generalized theory of elasticity to structural analysis.

Tensile testing is one of the most common ways of measuring material strength. It involves the linear stretching of a material until failure or some critical value is achieved.

\section{Analysis Obtained:}

- Tensile Strength

- Elongation at Break

\section{Equipment Required:}

- Universal Testing Machine(Tensile Testing Machine)

- Extensometer

- Data Acquisition

- Tensile Grips

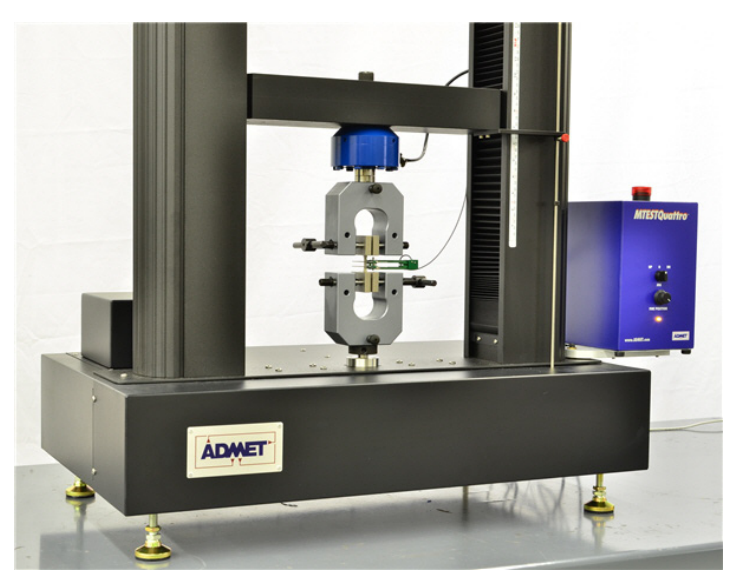

Figure 5:- Tensile Tester

\section{Result and Discussion}

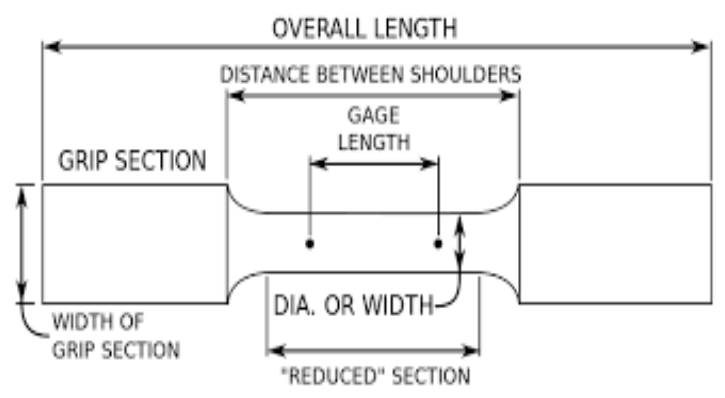

Figure 6:- Tensile Bar Configuration

This is the standard specimen size for the tensile test. The test continued until the failure of the specimen occurs. The Tensile strength obtained is in terms of Mpa. The three samples were tested by varying the percentage of RPP from $10 \%$ to $30 \%$ and the variations in the results were obtained and is compared with the VPP property.

Elongation at Break is the elongation corresponding to the point of rupture. Same standard ASTM D638 instrument and same standard specimens were used to found out the elongation at break and is basically expressed in percentage $(\%)$. 
Flexural strength Tester:- The Flexural strength of a material is defined as its ability to resist deformation under load. For materials that deform significantly but do not break, the load at yield, is typically measured at $5 \%$ deformation/ strain of the outer surface, is reported as flexural strength or flexural yield strength. The test specimen is under compressive stress at the concave surface and tensile stress at the convex surface.

The Flexural ASTM D790 Test that measures the force required to bend a beam under three point loading conditions. The data is often used to select the materials for parts that will support load without flexing. Flexural modulus is used as an indication of material's stiffness when flexed.

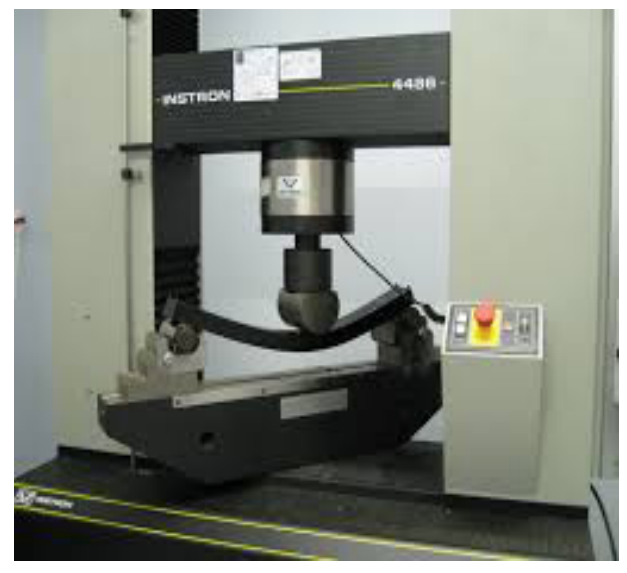

Figure 7:- Flexural 3 point tester

Izod Impact Strength:- Izod Impact Testing is an ASTM standard method of determining the impact resistance of materials. An arm held at a specific height (Constant Potential Energy) is released. The arm hits the sample. The specimen either breaks or the weight rests on the specimen. From the energy absorbed by the sample, its impact energy is determined. A notched sample is generally used to determine the impact energy and notch sensitivity.

The ASTM International standard for Izod Impact testing of plastics is D256. The results are expressed in energy lost per unit of thickness at the notch. Alternatively, the results may be reported as energy lost per unit cross- sectional area at the notch.

Impact is a very important phenomenon in governing the life of the structure. Impact tests are used in studying the toughness of the material. A material's toughness is a factor of its ability to absorb the energy during plastic deformation. The Impact value of the materials also changes with the temperature.

An impact is a high force or shock applied over a short time period when two or more bodies collide. Such as force or acceleration usually has a greater effect than a lower force applied over a proportionally longer period. The effect critically depends on the relative velocity of the bodies to one another.Road traffic accidents usually involve impact loading, when a car hits a traffic bollard, the damage being localized to the impact zone. When vehicle collide, the damage is proportionate to the relative velocity of the vehicles, the damage increasing as the square of the velocity since it is the impact kinetic energy which is the variable of importance. Much design effort is made to improve the impact resistance of cars so ass to minimize user injury. It can be achieved in several ways.

Izod Impact strength (Notched):- Notched Izod Impact is a single point test that measures a material resistance to impact from swinging 
pendulum. Izod Impact is defined as the kinetic energy needed to initiate fracture and continue fracture until the specimen is broken. Izod specimens are notched to prevent deformation of the specimen upon impact. This test can be used as a quick and easy quality control check to determine if a material meets specific impact properties to compare materials for general toughness.

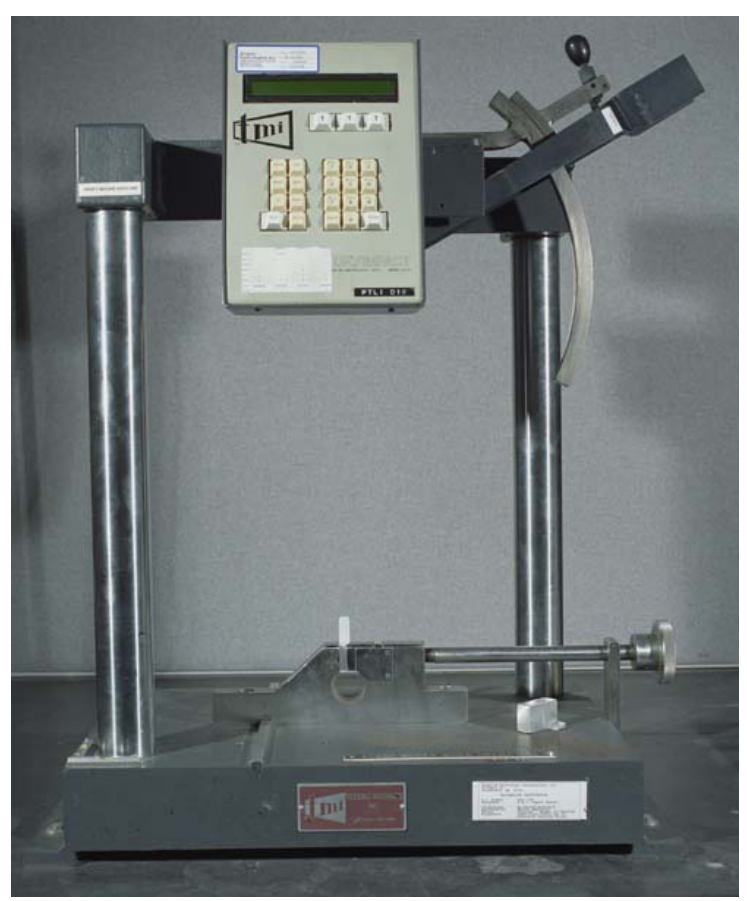

Figure 8:- Izod Impact (Notched)

\section{Heat Deflection Temperature (HDT):-} Heat Deflection Temperature is defined as the temperature at which a standard test bar deflects a specified distance under a load. It is used to determine short- term heat resistance. It distinguishes between materials that are able to sustain light loads at high temperatures and those that lose rigidity over a narrow temperature range.

The heat deflection temperature or heat distortion temperature is the temperature at which a polymer or plastic sample deforms under a specified load. This property of a given plastic material is applied in many aspects of product design, engineering, and manufacture of the products using thermoplastic components. The heat deflection temperature is determined by the ASTM D648 Test. The test specimen is loaded in three- point bending in the edgewise direction. The outer fiber stress used for testing is either $0.455 \mathrm{Mpa}$ or $1.82 \mathrm{Mpa}$ but we used is $0.455 \mathrm{Mpa}$, and the temperature is increased at $2^{\circ} \mathrm{c} / \mathrm{min}$ until the specimen deflects $0.25 \mathrm{~mm}$. This is similar to the test procedure defined in the ISO 75 standard.

Figure 1:- Bucket and Silicon oil gallon

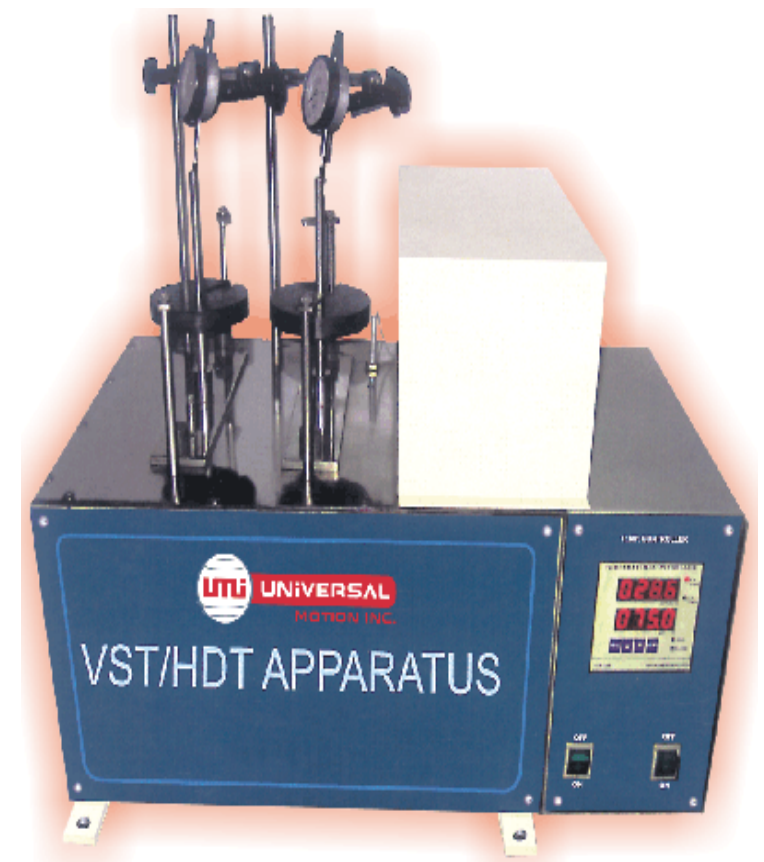

Figure 9 :- HDT Apparatus 
Original Data of VPP:

\begin{tabular}{|l|l|l|l|l|}
\hline S1. No. & Property & Standard & Unit & Results Obtained \\
\hline 1. & Tensile Strength@50mm/min & ASTM D638 & Mpa & 24.1318 \\
\hline 2. & Elongation at Break & ASTM D638 & $\%$ & $1.5-80$ \\
\hline 3. & Flexural Strength@ $1.44 \mathrm{~mm} / \mathrm{min}$ & ASTM D790 & $\mathrm{Mpa}$ & 37.23 \\
\hline 4. & Izod Impact Strength(Notched) & ASTM D256 & $\mathrm{KJ} / \mathrm{m}^{2}$ & 4.206 \\
\hline 5. & Heat Deflection Temperature $@ 0.45 \mathrm{MPa}$ & ASTM D648 & ${ }^{0} \mathrm{C}$ & 120 \\
\hline
\end{tabular}

Table 1:Mechanical properties data of VPP.

Tested Results and Comparison with VPP:

\begin{tabular}{|c|c|c|c|c|c|c|c|}
\hline \multirow{2}{*}{$\begin{array}{l}\text { S. } \\
\text { N. }\end{array}$} & \multirow[t]{2}{*}{ Property } & \multirow[t]{2}{*}{ Standard } & \multirow[t]{2}{*}{ Unit } & \multirow[t]{2}{*}{ VPP } & \multicolumn{3}{|c|}{ Results Obtained (By weight) } \\
\hline & & & & & $\begin{array}{l}\text { PP } 70 \% \text { with } \\
\text { EPDM } 10 \% \\
\mathrm{CaCO}_{3} 10 \% \\
\mathrm{RPP} 10 \%\end{array}$ & $\begin{array}{l}\text { PP } 60 \% \text { with } \\
\text { EPDM } 10 \% \\
\mathrm{CaCO}_{3} 10 \% \\
\text { RPP } 20 \%\end{array}$ & $\begin{array}{l}\text { PP } 50 \% \text { with } \\
\text { EPDM } 10 \% \\
\mathrm{CaCO}_{3} 10 \% \\
\mathrm{RPP} 30 \%\end{array}$ \\
\hline 1. & Tensile Strength@50mm/min & ASTM D638 & Mpa & 24.1318 & 20.4 & 18.2 & 20.7 \\
\hline 2. & Elongation at Break & ASTM D638 & $\%$ & $1.5-80$ & 73.0 & 65.5 & 79.8 \\
\hline 3. & FlexuralStrength@1.44mm/min & ASTM D790 & Mpa & 37.23 & 32.1 & 31.5 & 36.0 \\
\hline 4. & Izod Impact Strength(Notched) & ASTM D256 & $\mathrm{KJ} / \mathrm{m}^{2}$ & 4.206 & 4.40 & 5.98 & 4.34 \\
\hline 5. & $\begin{array}{l}\text { Heat Deflection Temperature@ } \\
0.45 \mathrm{MPa}\end{array}$ & ASTM D648 & ${ }^{0} \mathrm{C}$ & 120 & 114.2 & 112.3 & 126.0 \\
\hline
\end{tabular}

Table 2:Mechanical properties data of VPP and RPP with different proportions of VPP

\section{Graphs Section:}

Sample1: PP70\% with EPDM 10\%, $\mathrm{CaCO}_{3}$ $10 \%$ and RPP $10 \%$

Sample2: PP60\% with EPDM 10\%, $\mathrm{CaCO}_{3}$ $10 \%$ and RPP $20 \%$

Sample3: PP50\% with EPDM 10\%, $\mathrm{CaCO}_{3}$ $10 \%$ and RPP $30 \%$

\section{* Tensile Strength:}

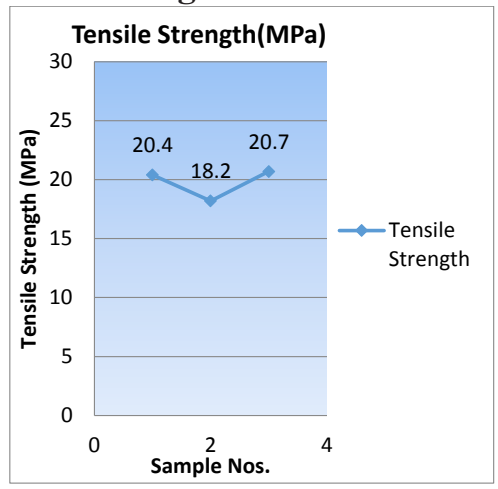

Graph 1:- Tensile Strength V/S Sample Nos.
* Elongation at Break:

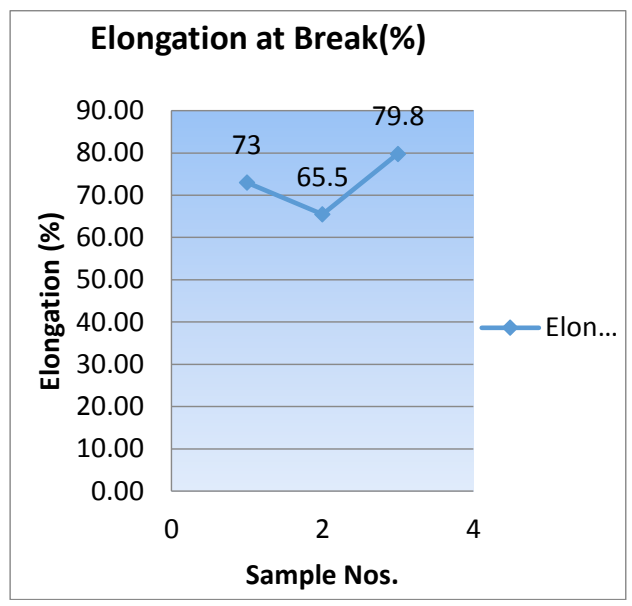

Graph 2:- Elongation at Break V/S Sample Nos.

\section{Flexural Strength:}




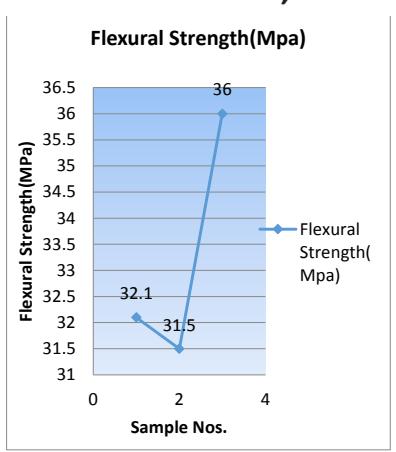

the proportions of Additives and Virgin Polypropylene as per the required mechanical and chemical properties. Whereas much more research needs to be done before using in aeronautical and aerospace industries where the materials are subjected to high pressure. The use of recycled polypropylene may reduce the burden of plastic pollution.

Graph 3:- Flexural Strength V/S Sample Nos.

* Izod Impact Strength (Notched):

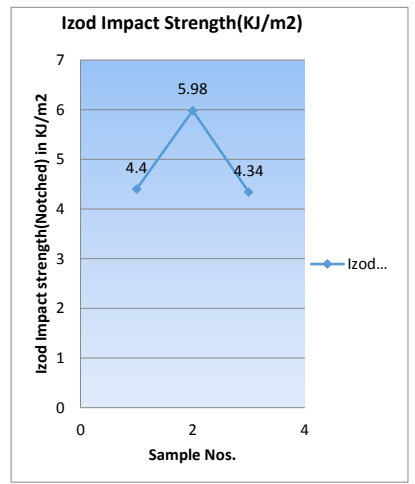

Graph 4:- Izod Impact Strength V/S Sample Nos.

\section{* Heat Deflection Temperature (HDT):}

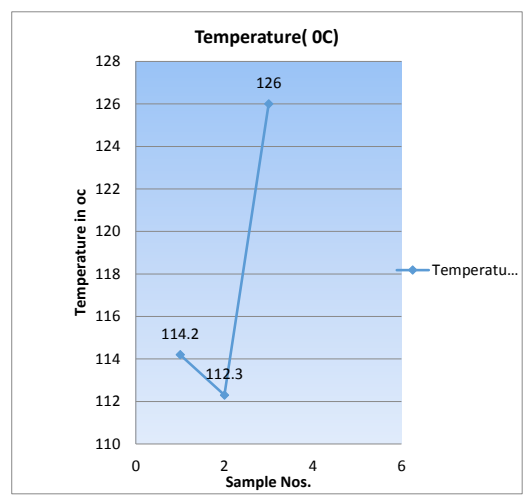

Graph 5:- Heat Deflection Temperature V/S

Sample Nos.

\section{CONCLUSION}

To reduce the plastic pollution for sustainable environmental balance Recycled Polypropylene can be used in different industries altering

\section{References}

[1] P. L 'ena, "Le plastique, toujours plus fantastique," Info Chimie Magazine,vol.42,no. 463,p.44,2005.

[2] "A.N.P.A. (Agenzia nazionale per la protezione dell'ambiente, O.N.R.(OssevatorioNazionaled eiRifiuti)Irifiutidelcomparto automobilistico," Studiodisettore, 2002.

[3] M. P. Luda, G. Ragosta, P. Musto et al., "Natural ageing of automotive polymer components: characterisation of new and used poly(propylene) based car bumpers,"Macromolecular MaterialEngeeneri ng,vol.287,no.6,pp.404-411,2002.

[4] Makuta, M., Moriguchi, Y., Yasuda, Y. and Sueno, S., Evaluation of the effect of automotive bumper recycling by life- cycle inventory analysis. J. Mater Cycle Waste Manage. 2(2000) 125-137

[5] $\wedge$ ab An introduction to the Rock- Forming Minerals, 2 ed., by W.A. Deer, R.A. Howie, and J. Zussman, 192 Prentice Hall, ISBN 0-582-30094-0.

[6] Jalilvand AR, Ghasemi I, Karrabi M, Azizi $\mathrm{H}$, using devulcunized EPDM in PP/HDPE/ EPDM ternary blend, mechanical properties and morphology, iran polym J,16, 637-644, 2007.

[7] Purnima D, Maiti SN, Gupta AK, Interfacial adhesion through maleic anhydride grafting of EPDM in PP / EPDM blend, J Appl Polym Sci, 102, 5528-5532, 2006.

[8] Testing Facility : Central Institute of Plastic Engineering and Technology, Government office in Chennai, India 